\title{
Robust Disturbance-Force Compensator for Time Waveform Replication of an Electrodynamic Shaker*
}

\author{
Yasuhiro UCHIYAMA** and Masayuki FUJITA*** \\ ** IMV CORPORATION \\ 2-6-10 Takejima, Nishiyodogawa-ku, Osaka 555-0011, Japan \\ E-mail: uchiyama@imv.co.jp \\ *** Department of Mechanical and Control Engineering, Tokyo Institute of Technology \\ 2-12-1 Ookayama, Meguro-ku, Tokyo 152-8550, Japan
}

\begin{abstract}
This paper presents a disturbance-force compensator for an electrodynamic shaker. The characteristics of a shaking system are considered to be nonlinear and variable because of the influence of the test piece. In order to compensate for this problem, the influence of the disturbance force needs to be suppressed. The controller is designed using $\mu$-synthesis by considering the uncertainty of the shaker. In order to investigate the control performance in relation to the influence of friction and sloshing, time waveform replication testing is executed. Finally, because the compensator suppresses nonlinearity, a good performance can be realized, as confirmed by experiments conducted using actual equipment.
\end{abstract}

Key words : Robust Control, Vibration Control, Control Applications, $\mu$-synthesis, Disturbance Compensator, Time Waveform Replication, Electrodynamic Shaker

\section{Introduction}

In order to confirm the durability of the specimens, shakers are used in vibration tests. The specimen is a test piece, and it is exposed to a vibration environment under actual field conditions in the vibration test. Recently, shaking systems that can more accurately replicate an actual situation have been widely employed for vibration testing. Shaking systems are mainly used in the automotive industry. They are also used in the civil and architectural engineering fields. An electrodynamic shaker has some positive features such as good linearity and a wide frequency response. The shaking system needs to be controlled not only for exercising stable control but also for ensuring better replication of the given reference waveform. However, if the interaction between the shaker and the test piece cannot be ignored, it will be difficult to control the shaking system ${ }^{(1)}$. Further, if the characteristics of a controlled plant are nonlinear because of the influence of the test piece, control becomes difficult.

Many applications have been developed to realize robust vibration control, for example, control for sloshing suppression ${ }^{(2)}$, control of dual-stage actuators for hard disk drives ${ }^{(3)}$, control of an active suspension ${ }^{(4)}$, stability analysis for mechanical vibration systems ${ }^{(5)}$, and pointing control of mobile antennas using $H_{\infty}$ gain-scheduled controller synthesis ${ }^{(6)}$. In our previous studies $^{(7)}$, good performance was achieved using a robust feedback controller. However, it was assumed that the test piece was invariant and the perturbation of the controlled plant was small. Therefore, if plant perturbation becomes significant, the control performance cannot satisfy the test specifications, although the system stability can be compensated.

With regard to the problems of the abovementioned control, for example, the real-time compensation of the reaction force to eliminate the influence of the specimen ${ }^{(8)(9)}$, and MCS algorithm, one of the model reference adaptive control algorithms, has been successfully ap-

*Received 17 Aug., 2009 (No. 09-0455) [DOI: 10.1299/jsdd.4.1]

Copyright (C) 2010 by JSME 


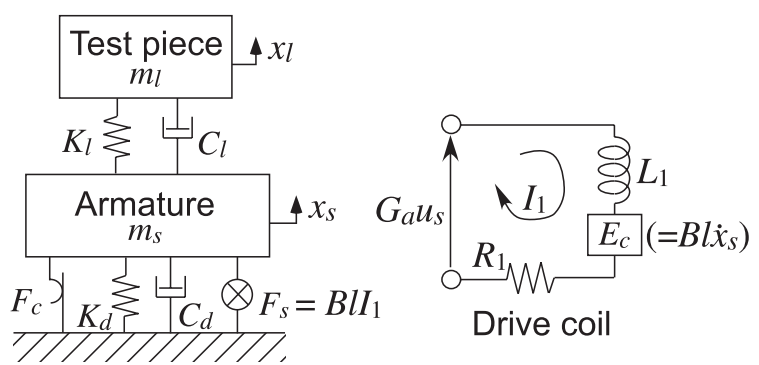

Fig. 1 Schematic diagram of the shaker model and equivalent circuit.

plied $^{(10)}$. In particular, Ref. (8) applied the control with disturbance observer-based compensation for the reaction force in the specimen. However, although this method provided good results, uncertainties were not explicitly considered. Another study proposed a control that guaranteed the robust stability of the disturbance observer for hard disk servo systems ${ }^{(11)}$, and the control was also applied to a xy feed table ${ }^{(12)}$. In this study, difficulties are encountered in measuring the disturbance force directly, and it is therefore necessary to use the estimated values of the force. The disturbance force is then estimated using the displacement, acceleration, and current responses; these three variables have been employed in order to reduce noise and obtain an accurate value. Nevertheless, it is evident that uncertainties need to be considered due to the influence of the difference between the nominal model and the actual plant.

For stable control, the design process takes into account the the perturbation of parameters used in estimating the disturbance-force. The estimator is also added to a generalized plant, and the controller is then designed by $\mu$-synthesis. Furthermore, the replication performance of the proposed control system is evaluated for the abovementioned problem. The disturbance-force compensator is designed to suppress the influence of the specimen and the nonlinear characteristics, and a simple open-loop controller is employed to replicate the reference signal. Owing to the advantage of using the disturbance-force compensator, it is considered that the proposed control structure provides a good control performance regardless of whether a simple open-loop controller is employed.

The rest of the paper is organized as follows: The control problem is described in Section 2; the mathematical model and the uncertainty weighting function for the proposed compensator are introduced in Section 3; the design of the feedback controller using $\mu$-synthesis is discussed in Section 4; experimental results for sloshing in a liquid container are discussed in Section 5; and finally the paper is summarized in Section 6.

\section{Control problem}

The characteristics of a controlled plant are nonlinear and variable because of the influence of the test piece. Consequently, control is difficult. This problem occurs because the disturbance force added to the shaking system is induced by the interaction between the shaker and the test piece. When the characteristics of the controlled plant change, the influence of this problem becomes significant. Other forces such as friction are also included in this disturbance force.

In this study, our aim is to compensate for this problem by suppressing the influence of the disturbance force. For instance, let us consider a simple shaker model that comprises a test piece exhibiting resonance and takes into account the influence of friction on the armature, as shown in Fig. 1. The dynamic equations of the shaker model are

$$
\begin{aligned}
& F_{s}=m_{s} \ddot{x}_{s}+C_{d} \dot{x}_{s}+K_{d} x_{s}+d_{f} \\
& d_{f}=C_{l}\left(\dot{x}_{s}-\dot{x}_{l}\right)+K_{l}\left(x_{s}-x_{l}\right)+F_{c} \\
& 0=m_{l} \ddot{x}_{l}-C_{l}\left(\dot{x}_{s}-\dot{x}_{l}\right)-K_{l}\left(x_{s}-x_{l}\right),
\end{aligned}
$$

where $F_{s}$ denotes the electrodynamic force; $F_{c}$, the friction force; and $d_{f}$, the disturbance force. $x_{s}$ and $x_{l}$ respectively denote the displacement of the armature and the specimen; $K_{d}$ and $C_{d}$, the stiffness and damping coefficient of the suspension system of the shaker; $K_{l}$ and 
$C_{l}$, the stiffness and damping coefficient of the specimen; and $m_{s}$ and $m_{l}$, the masses of the armature and the specimen.

According to Eq. (2), $d_{f}$ comprises the influences of friction on the armature and resonant characteristics of the specimen. In order to suppress these influences, an electrodynamic force $d_{s}$ is generated to counteract $d_{f}$ using the proposed controller and is applied as follows:

$$
F_{s}=F_{r}-d_{s},
$$

where $F_{r}$ denotes the electrodynamic force used in the tracking control of the reference signal. If it is assumed that $d_{s} \equiv-d_{f}$, the influence of $d_{f}$ is then suppressed.

When the controller is designed to compensate for such influences, knowledge about the test piece model and friction characteristics is required. However, if it is assumed that $d_{f}$ can be measured as a controlled variable, the control system can be constructed without introducing the characteristics that induce $d_{f}$. The objective of this study is to design a disturbance-force compensator that generates such a $d_{s}$, provided the characteristics that induce the disturbance force are not considered.

\section{Model of the electrodynamic shaker}

\subsection{Nominal model}

The electrodynamic shaker is based on the principle that an electrodynamic force is generated in proportion to the current applied to the drive coil existing in the magnetic field. Armature displacement and acceleration are measured using a laser displacement sensor and piezoelectric accelerometer, respectively. The current of the drive coil is measured using a current transducer. Assuming that the magnetic flux density is constant, a drive coil can be shown as a linear equivalent circuit in Fig. 1.

The force $F_{s}$ and the reverse electromotive force $E_{c}$ can be represented as

$$
\begin{aligned}
& F_{s}=B l I_{1} \\
& E_{c}=B l \dot{x}_{s},
\end{aligned}
$$

where $I_{1}$ denotes the current of the drive coil; $l$, the length of the drive coil; and $B$, the magnetic flux density. From Fig. 1, the following Eq. (7) can be obtained

$$
G_{a} u_{s}=R_{1} I_{1}+L_{1} \dot{I}_{1}+E_{c}
$$

where $G_{a}$ and $u_{s}$ denote the amplifier gain and the input voltage to the amplifier, respectively. As stated above, the mathematical model of the electrodynamic shaker is obtained using Eqs. (1) and (5)-(7).

In order to add the nonlinear characteristic of the shaker, friction is generated on the armature by increasing the clamping pressure of the linear motion guide device. The input voltage level exhibits nonlinearity in the frequency response. Models that correspond to an input voltage can be identified by altering the parameters of the suspension system. The model that is measured by using white noise, set at $80 \mathrm{mV} \mathrm{rms}$, is defined as the nominal model. Because the accurate measurement of values is difficult due to the influence of friction, it is necessary to use a controller in which the uncertainties are explicitly considered.

\subsection{Estimation of disturbance force}

It is difficult to measure the disturbance force directly. In this study, the disturbance force is estimated using the displacement and acceleration responses of the armature and the current response of the drive coil. To reduce the influence of noise and obtain accurate estimated values, the order of the estimator needs to be low. Therefore, three variables are employed. A schematic diagram for estimating the disturbance force is depicted in Fig. 2. As shown in this figure, the estimated disturbance force $\hat{d}_{f}$ is given by

$$
\begin{aligned}
& \hat{d}_{f}=G_{E}\left[\begin{array}{lll}
I_{1} & \ddot{x}_{s} & x_{s}
\end{array}\right]^{T} \\
& G_{E}=\left[\begin{array}{lll}
B l & -\left(m_{s}+\frac{C_{d}}{s+\omega_{p}}\right) & -K_{d}
\end{array}\right],
\end{aligned}
$$




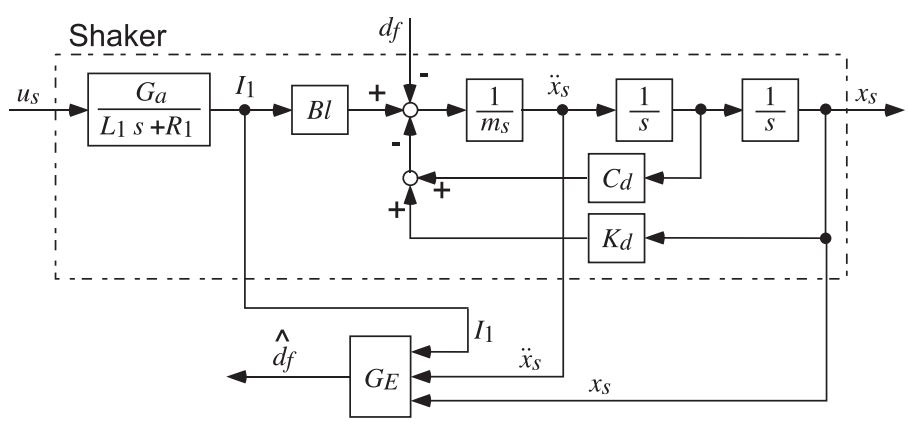

Fig. 2 Schematic diagram of the shaker and the disturbance estimator.

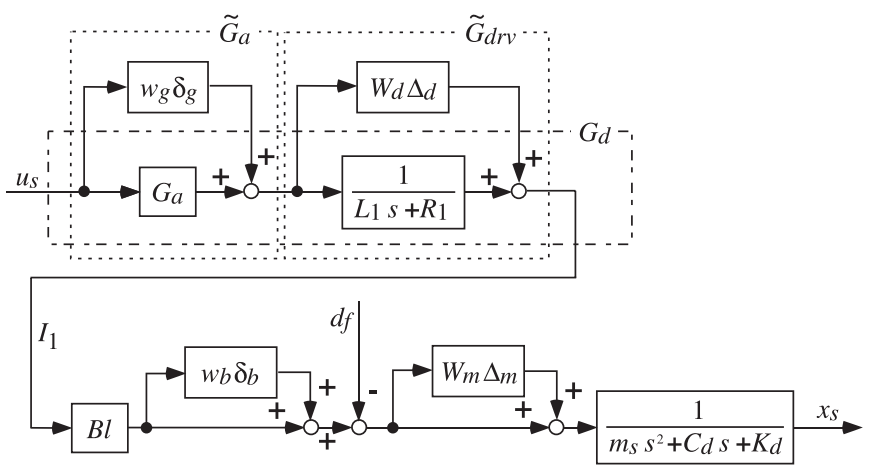

Fig. 3 Schematic diagram of perturbation structure.

where $\omega_{p}$ denotes an adjustment parameter to keep the gain low in a low frequency band.

It is difficult to express the precise characteristics of an actual plant using a mathematical model, and some uncertainties are generally present. Furthermore, since friction is generated on the armature, identification is more difficult. In order to realize robust control, perturbations in the parameters $m_{s}, K_{d}$, and $C_{d}$ need to be considered in the design. On the other hand, it is considered that these value mismatches are included in the estimated disturbance force. Thus, in compensating for the estimated disturbance force, this difference is small.

\subsection{Modeling uncertainty}

The perturbation structure being considered is shown in Fig. 3. The range of perturbation of each parameter is set as shown in Table 1. When the equivalent circuit of the drive coil is considered, the transfer function from the input voltage $E$ to the current $I_{1}$ is represented as $1 /\left(L_{1} s+R_{1}\right)$. It is difficult to describe this characteristic accuratly in the model, and perturbation is considered as an unstructured additive uncertainty. The frequency responses of these additive perturbations in the drive coil are plotted by using the minimum and maximum values of each parameter in Fig. 4. The magnitude of the uncertainty weighting function $W_{d}$ is selected to cover all of the perturbed models as follows ${ }^{(13)}$ :

$$
W_{d}=0.11 \cdot \frac{s+500 \cdot 2 \pi}{s+100 \cdot 2 \pi} .
$$

The additive perturbation for the amplifier gain is represented as

$$
\tilde{G}_{a}=G_{a}+w_{g} \delta_{g}, \quad \delta_{g} \in[-1,1]
$$

where $\tilde{G}_{a}$ and $w_{g}$ denote the actual parameter and the weighting coefficient, respectively. The multiplicative output perturbation of the magnetic flux density is represented as

$$
\tilde{B}=\left(1+w_{b} \delta_{b}\right) B, \quad \delta_{b} \in[-1,1],
$$

where $\tilde{B}$ and $w_{b}$ denote the actual parameter and the weighting coefficient, respectively. 
Table 1 Ranges of perturbed parameters for the electrodynamic shaker.

\begin{tabular}{|c|c|}
\hline Parameter & Range of perturbation \\
\hline$R_{1}$ & -10 to $10 \%$ \\
$L_{1}$ & -15 to $15 \%$ \\
$B$ & -10 to $10 \%$ \\
$G_{a}$ & -5 to $5 \%$ \\
$K_{d}$ & -10 to $10 \%$ \\
$C_{d}$ & -12 to $12 \%$ \\
$m_{s}$ & -9 to $9 \%$ \\
\hline
\end{tabular}
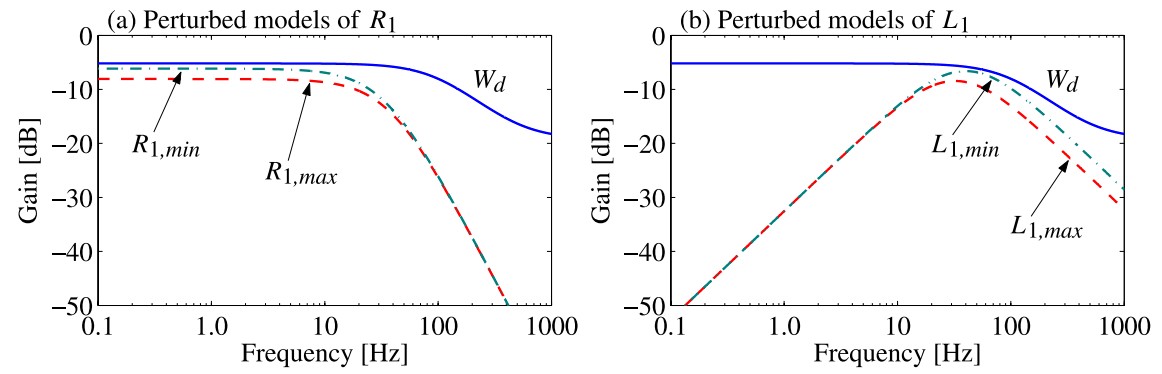

Fig. 4 Weighting function $W_{d}$ (solid line) and additive perturbations: (a) resistance $R_{1}$ and (b) inductance $L_{1}$. The perturbed models derived by using the maximum values are indicated by the dashed red line. The perturbed models derived by using the minimum values are indicated by the chained green line.

Because accurate identification of each parameter in Eq. (8) is difficult, the uncertainties in the mechanical component of the model need to be considered. The transfer function of this component is represented as $1 /\left(m_{s} s^{2}+C_{d} s+K_{d}\right)$, and the multiplicative output uncertainty caused by parameter perturbation is considered using a simple control structure in the next section. It is assumed that this uncertainty is represented as an unstructured uncertainty, and the perturbed models of parameters $m_{s}, K_{d}$, and $C_{d}$ are plotted in Fig. 5. The magnitude of the uncertainty weighting function $W_{m}$, selected to cover all the perturbations, can be expressed as follows:

$$
W_{m}=0.009 \cdot \frac{s+3 \cdot 2 \pi}{s+7 \cdot 2 \pi} \cdot \frac{s+300 \cdot 2 \pi}{s+7 \cdot 2 \pi} .
$$

\section{Controller design}

\subsection{Control objectives}

The design of the disturbance-force compensator of an electrodynamic shaker is carried out using MATLAB. The following items are set as control objectives.

- Stabilize the system even when an uncertainty exists.

- Maintain performance wherein the disturbance force is sufficiently suppressed.

A controller is designed to maintain robust stability and performance against the uncertainty model. The controller for this system is then designed by $\mu$-synthesis, which has many applications, such as Ref. (13). In order to replicate the reference waveform, a simple open-loop control method is used. In this method, the control input is calculated from the measurement transfer function; only the input signal is employed, and it is not varied in the vibration test. The control error increases when there is a difference between the transfer function and the actual characteristics. Therefore, this mismatch needs to be reduced using the disturbance-force compensator.

Let us consider the feedback structure shown in Fig. 6, where block $K_{f}$ corresponds to the disturbance-force compensator. In order to consider a simple control structure, it is also assumed that the disturbance force is estimated by using the displacement and current signal in this design section; $\hat{d}_{f}$ is given instead of Eq. (8) as follows

$$
\hat{d}_{f}=G_{E}^{\prime}\left[\begin{array}{ll}
I_{1} & x_{s}
\end{array}\right]^{T}
$$



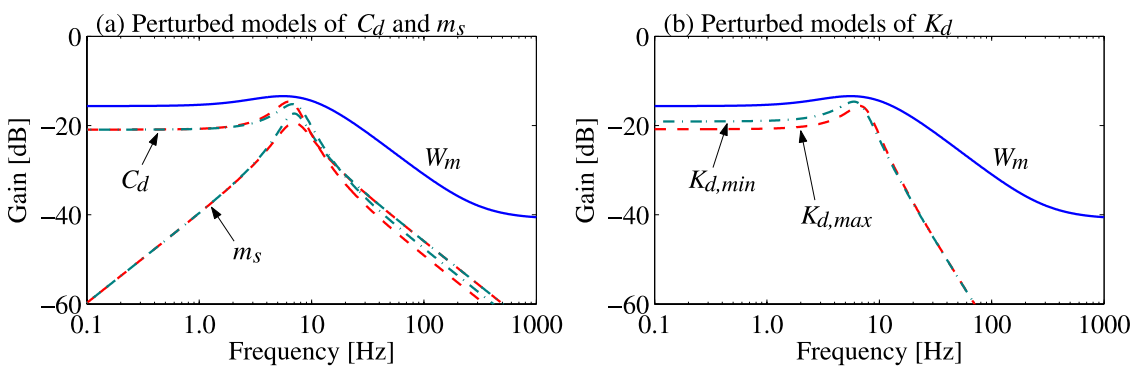

Fig. 5 Weighting function $W_{m}$ (solid line) and multiplicative output perturbations: (a) damping coefficient $C_{d}$ and armature mass $m_{s}$ and (b) stiffness coefficient $K_{d}$. The perturbed models derived by using the maximum values are indicated by the dashed red line. The perturbed models derived by using the minimum values are indicated by the chained green line.

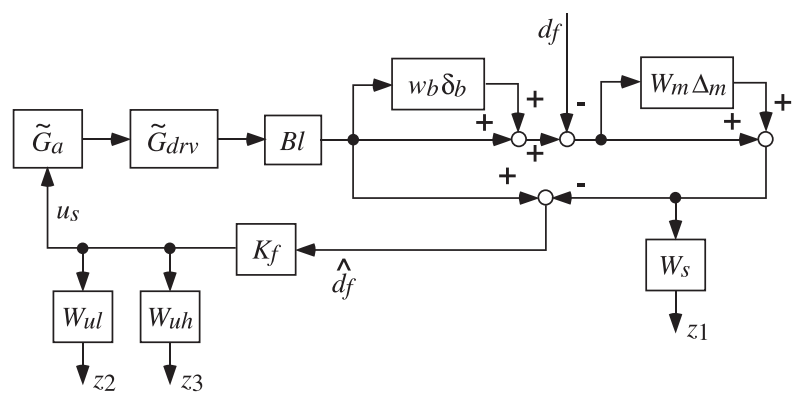

Fig. 6 Feedback structure used for designing the disturbance-force compensator.

$$
G_{E}^{\prime}=\left[\begin{array}{ll}
B l & -\left(\frac{1}{m_{s} s^{2}+C_{d} s+K_{d}}\right)^{-1}
\end{array}\right] .
$$

As shown in Fig. 6, because the nominal model of the mechanical component is counterbalanced by the second element of $G_{E}^{\prime}$, the structure is represented as a simple model that is constructed using only the perturbed blocks.

\section{2. $\mu$-synthesis}

The weighting function $W_{s}$ is considered for the suppression of the disturbance force. To improve the performance, the gain of the control frequency band needs to be enlarged within achieving robust stability. Accordingly, $W_{s}$ is now expressed as

$$
\begin{aligned}
W_{s}= & 2.9 \cdot \frac{14 \cdot 2 \pi}{s+14 \cdot 2 \pi} \cdot \frac{70 \cdot 2 \pi}{s+70 \cdot 2 \pi} \cdot \frac{140 \cdot 2 \pi}{s+140 \cdot 2 \pi} \\
& \cdot \frac{s}{s+2.4 \cdot 2 \pi} \cdot \frac{s}{s+0.56 \cdot 2 \pi} .
\end{aligned}
$$

The frequency response of $W_{s}$ is shown in Fig. 7 (a).

In practice, the measurement of signals is affected by noise. Since the signal-to-noise ratio of the displacement response signal is low at high frequency, the magnitude of the controller needs to be small at high-frequency bands. The weighting function $W_{u h}$ is then expressed as

$$
W_{u h}=2000 \cdot \frac{s+2.5 \cdot 2 \pi}{s+4000 \cdot 2 \pi} .
$$

On the other hand, since the signal-to-noise ratio of the acceleration response signal is low at low frequency, the magnitude of the controller must be small at low-frequency bands. The weighting function $W_{l l}$ is then expressed as

$$
W_{u l}=0.9 \cdot \frac{s^{2}+2.5 \cdot 2 \pi \sqrt{2} s+(2.5 \cdot 2 \pi)^{2}}{s^{2}+0.002 \cdot 2 \pi \sqrt{2} s+(0.002 \cdot 2 \pi)^{2}} .
$$



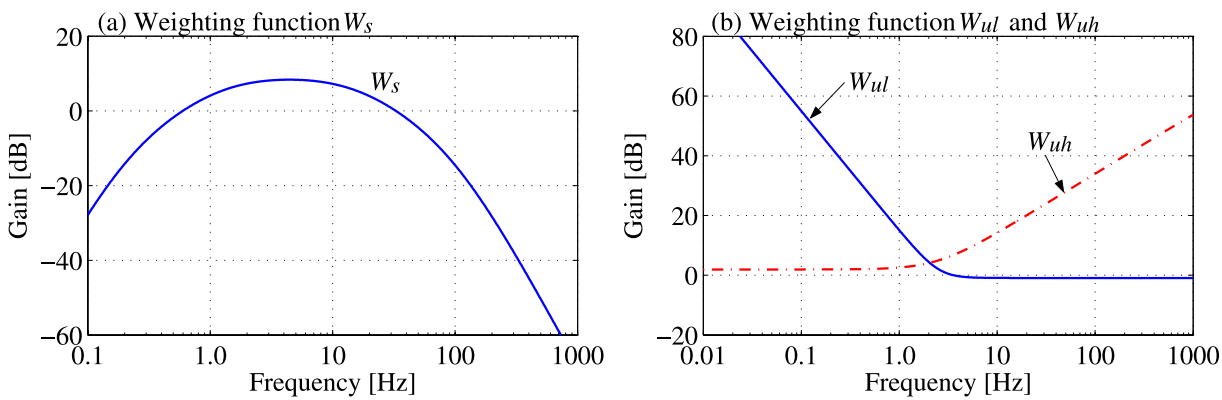

Fig. 7 Weighting function: (a) $W_{s}$ for the control error and (b) $W_{u l}$ (solid line) and $W_{u h}$ (chained line) for the control input.

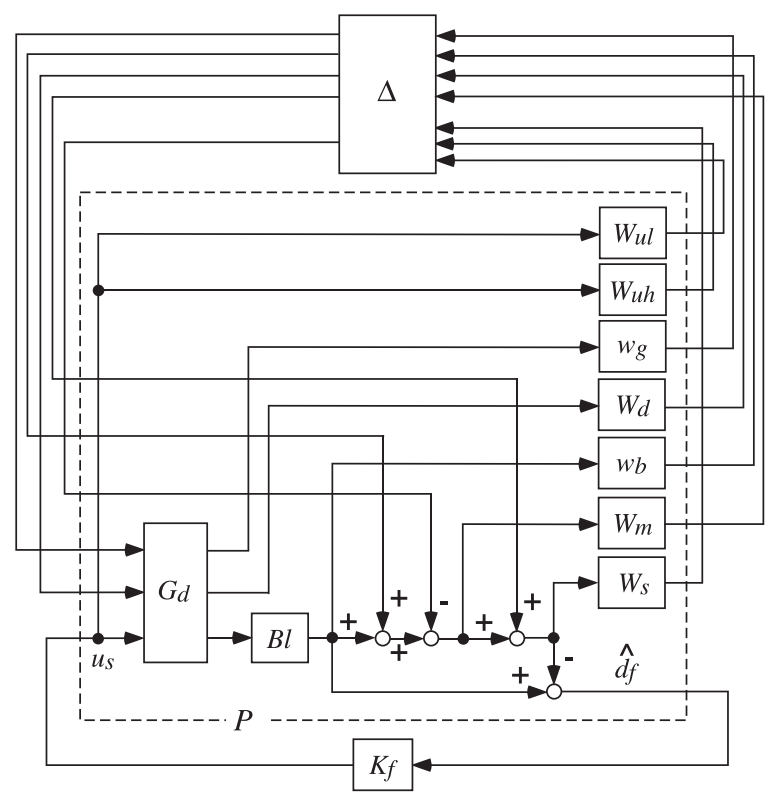

Fig. 8 Block diagram of generalized plant $P$ designed using $\mu$-synthesis.

The frequency responses of $W_{u h}$ and $W_{u l}$ are shown in Fig. 7 (b). It is noted that $W_{u h}$ and $W_{u l}$ are separated in order to simplify the adjustment of the weighting functions.

The design objective for stability and control performance is formalized as the requirement for a closed-loop transfer function with weighting functions. Therefore, the generalized plant $P$, which is shown in Fig. 8, is constructed to treat the control objectives of the $\mu$-synthesis framework. Here, the block structure of the uncertainty $\Delta$ is defined as

$$
\begin{aligned}
\Delta:= & \left\{\operatorname{diag}\left(\delta_{g}, \delta_{b}, \Delta_{d}, \Delta_{m}, \Delta_{p}\right), \delta_{g} \in C^{1 \times 1}, \delta_{b} \in C^{1 \times 1},\right. \\
& \left.\Delta_{d} \in C^{1 \times 1}, \Delta_{m} \in C^{1 \times 1}, \Delta_{p} \in C^{1 \times 3}\right\},
\end{aligned}
$$

where $\left|\delta_{g}\right| \leq 1,\left|\delta_{b}\right| \leq 1,\left|\Delta_{d}\right| \leq 1,\left|\Delta_{m}\right| \leq 1,\left\|\Delta_{p}\right\|_{\infty} \leq 1$, and $\Delta_{p}$ is a fictitious uncertainty block used for achieving robust performance. Next, it is considered that $P$ is partitioned as

$$
P=\left[\begin{array}{ll}
P_{11} & P_{12} \\
P_{21} & P_{22}
\end{array}\right] .
$$

From Fig. 8, the linear fractional transportation on $P$ by $K_{f}$ is defined as follows:

$$
F_{l}\left(P, K_{f}\right):=P_{11}+P_{12} K_{f}\left(I-P_{22} K_{f}\right)^{-1} P_{21} .
$$

The robust performance condition is equivalent to the structured singular value $\mu$ as follows.

$$
\sup _{\omega \in \mathcal{R}} \mu_{\Delta}\left(F_{l}\left(P, K_{f}\right)(j \omega)\right)<1
$$




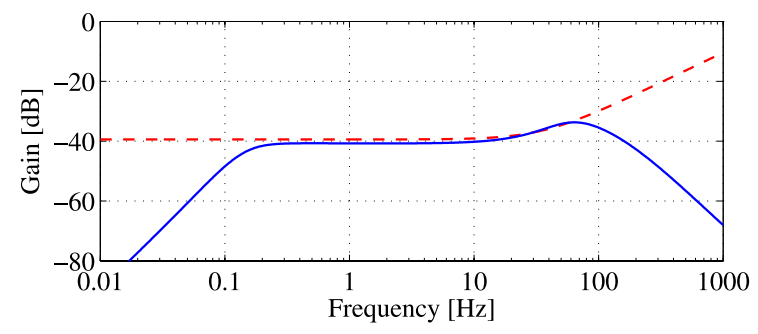

Fig. 9 Frequency responses of the disturbance-force compensator (solid line) and the ideal characteristic (dashed line).

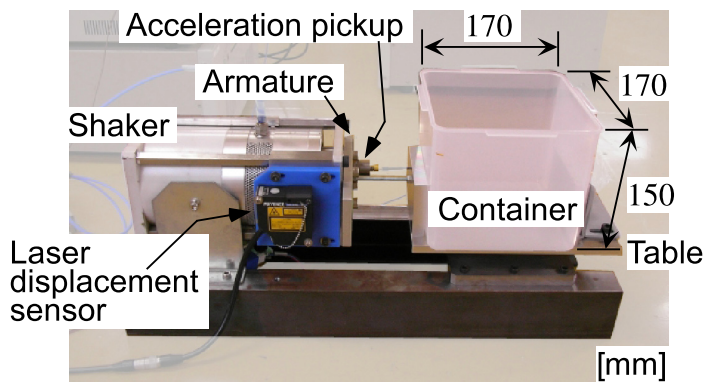

Fig. 10 Overview of experimental setup.

Since the controller satisfies this condition, the $\mathrm{D}-\mathrm{K}$ iteration procedure is employed. A controller that satisfies Eq. (20) is obtained after two iterations. The degree of this controller can be reduced from 47 states to 15 states. On the other hand, an ideal characteristic of the disturbance-force compensator, which is designed for the case in which uncertainties are excluded, can be represented by an inverse transfer characteristic from the input voltage $u_{s}$ to a summing point of force, which is expressed as $\left(L_{1} s+R_{1}\right) / G_{a} B l$. A comparison of the obtained compensator with the ideal characteristic is shown in Fig. 9. Each characteristic exhibits a similar tendency at the control frequency bands. In addition, the gain at low- and high-frequency bands decreases because $W_{u l}$ and $W_{u h}$ are considered for the influence of the noise.

\section{Control results}

\subsection{Experimental setting}

The control performance is confirmed by experiments using the system shown in Fig. 10. In order to enable the characteristics of a controlled plant to be changed, a covered container with water is employed as the specimen for the excitation experiment. Sloshing, which is liquid vibration, occurs when the container is excited. It is difficult to control in the resonant frequency of sloshing. The size of the rectangular container is $170 \mathrm{~mm} \times 170 \mathrm{~mm} \times 150 \mathrm{~mm}$ (length, width, height). If the liquid level is set higher than $80 \mathrm{~mm}$, the resonance is at approximately $2 \mathrm{~Hz}^{(2)}$.

The disturbance-force compensator is discretized using Tustin transform at a sampling frequency of $512 \mathrm{~Hz}$. In these experiments, the disturbance force is estimated using Eq. (8). First, to investigate the influence of the nonlinear characteristics, the transfer function is measured using of white noise input signals, the rms value of which differs correspondingly. As mentioned above, friction is generated on the armature. Therefore, using comparisons with each frequency response for the input voltage level, the control performance for nonlinear characteristics is evaluated.

Next, the control performance is evaluated by time waveform replication, and a sine waveform set at $2 \mathrm{~Hz}$ is used as the reference signal. A drive signal, which is a control input, is generated using open-loop control. The inverse characteristic of the transfer function is calculated, and the drive signal is obtained using the inverse characteristic and the reference signal. A study of sloshing is generally conducted in order to suppress it. However, in this 

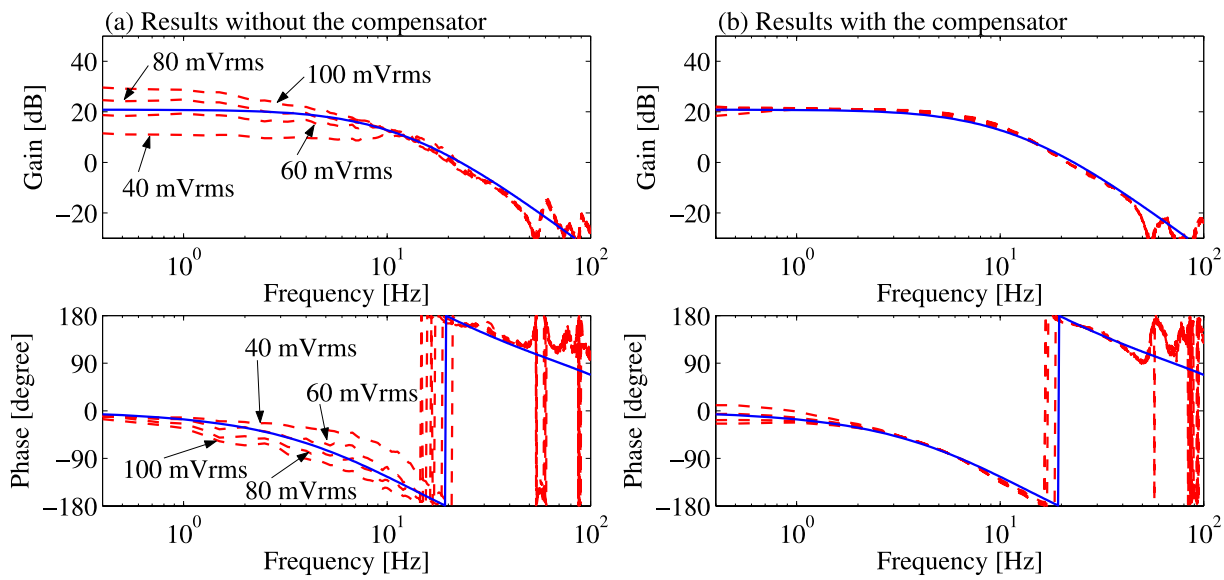

Fig. 11 Frequency responses of transfer functions: (a) results without the disturbanceforce compensator and (b) results with the compensator. The nominal model is indicated by the solid line. The measured transfer functions are indicated by dashed lines.

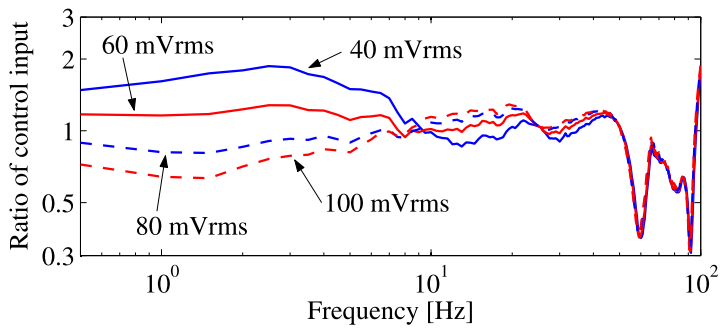

Fig. 12 Frequency characteristics of the ratio of control inputs with the disturbanceforce compensator to that without the compensator.

experiment, satisfactory replication is required irrespective of the influence of sloshing.

\subsection{Experimental results of frequency response}

The experiment is executed with and without the disturbance-force compensator. The liquid level in the container is set at $100 \mathrm{~mm}$. The rms value of the input voltage is increased from 40 to $100 \mathrm{mV} \mathrm{rms}$, and the transfer function from $u_{s}$ to $x_{s}$ is measured. The results without the controller are shown in Fig. 11 (a). For the frequency response, because each transfer function represents different characteristics, it is considered that friction is associated with nonlinear characteristics.

The results with the disturbance-force compensator are shown in Fig. 11 (b). Both the gain and the phase characteristic of each measured transfer function are consistent with the nominal model in the frequency band from $1 \mathrm{~Hz}$ to $10 \mathrm{~Hz}$. Because the disturbance-force compensator suppresses the influence of the nonlinear characteristics, good results are obtained. Furthermore, the frequency response of control inputs is evaluated. The frequency characteristics of the ratio of control inputs with the disturbance-force compensator to that without the compensator are calculated, and the results are shown in Fig. 12. When the rms value of the input voltage is small, the gain increases at low-frequency bands because the influence of friction becomes strict. In contrast, when a larger input voltage is used, the amplitude of the gain is small because the influence of friction decreases.

Therefore, in the case of time waveform replication using open-loop control, it is considered that the control performance with the disturbance-force compensator is better than that without the compensator.

\subsection{Experimental results of transient response}

The transient response is also confirmed in cases with and without the disturbance-force 
(a) Reference and response
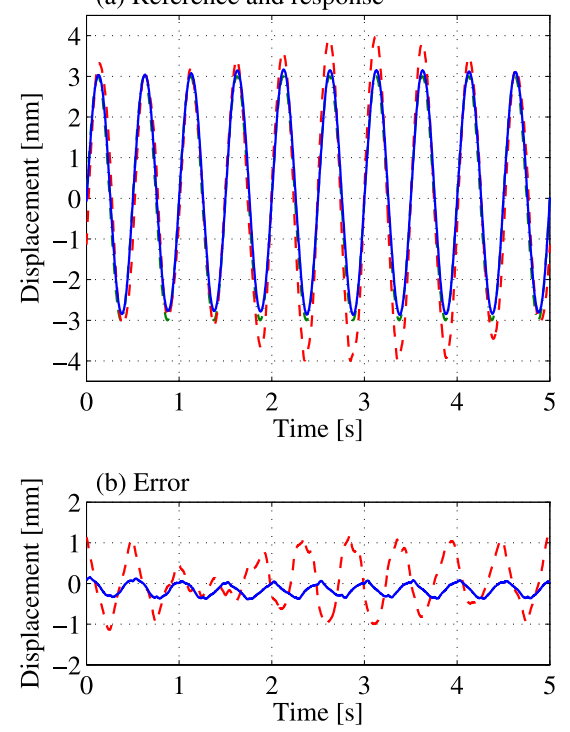

(c) Ref. and res. (Zoomed in 5 times)
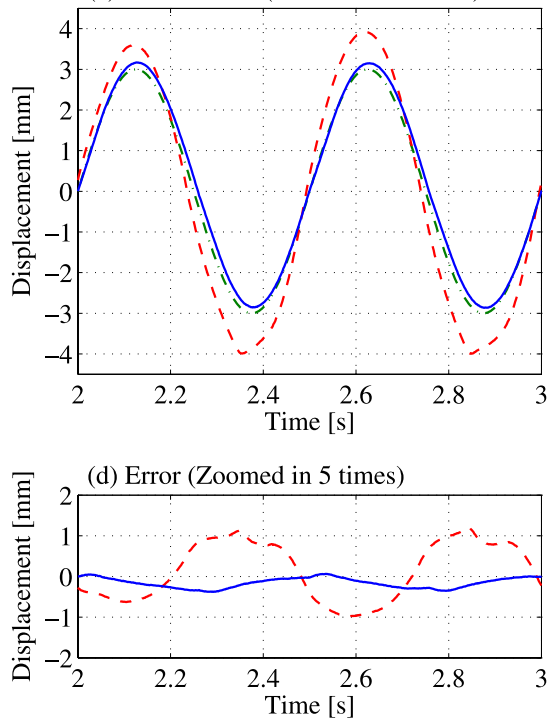

Fig. 13 Excitation results when the water level is set at $100 \mathrm{~mm}$ : (a, c) reference and response signals, and (b, d) error signal. (c) and (d) show signals with 5 times magnification. The reference signal is indicated by the dashed chained line. The response without the compensator is indicated by the dashed red line. The response with the compensator is indicated by the solid blue line.

compensator. Each drive signal is generated using the transfer functions set at $80 \mathrm{mV} \mathrm{rms} \mathrm{in}$ Fig. 11 (a) and (b), and these two drive signals are employed in every scenario. The amplitude level of the sine waveform used as the reference signal is set constant.

First, the liquid level is set at $100 \mathrm{~mm}$, and the transfer functions for generating the drive signal are measured under this condition. The control results are shown in Fig. 13 (a); the error signals, in Fig. 13 (b). The signals in Fig. 13 (c) and (d) are shown with 5 times magnification. As the result, regardless of the state being the same when the transfer function is measured, the result without the compensator is inconsistent with the reference signal. Since the response signal without the compensator is not a sine waveform with a constant level, it is considered that the nonlinear characteristics exist in the controlled system.

Next, the liquid level is set at $130 \mathrm{~mm}$, but the same drive signals and disturbance-force compensator are used. The control results are shown in Fig. 14. As in Fig. 13, the result without the compensator is inconsistent with the reference signal due to the influence of the nonlinear characteristics. Furthermore, a comparison of Fig. 14 with Fig. 13 reveals that each response without the compensator is different due to the change in the liquid level. However, although the characteristic of the controlled plant changes, the proposed method yields a good response signal that is consistent with the reference signal, thereby suppressing the influence of the nonlinear characteristics.

Furthermore, to investigate the transient influence of friction, an increasing sine is employed as the reference signal; the amplitude of the wave is increased from $0 \mathrm{~mm}$ to $3 \mathrm{~mm}$ over $3 \mathrm{~s}$. The liquid level is set at $100 \mathrm{~mm}$, and the drive inputs are calculated using the transfer functions of the previous experiment. The control results are shown in Fig. 15. When the disturbance-force compensator is not used, the influence of friction appears because the level of the reference signal is small for the time period up to $1.5 \mathrm{~s}$, and the error signal is then enlarged. On the other hand, the result with the disturbance-force compensator shows that good replication is achieved because the influence of the nonlinear characteristics is suppressed.

These results support the conclusion that the proposed method can yield satisfactory time waveform replication irrespective of whether or not the test piece model is introduced. 
(a) Reference and response
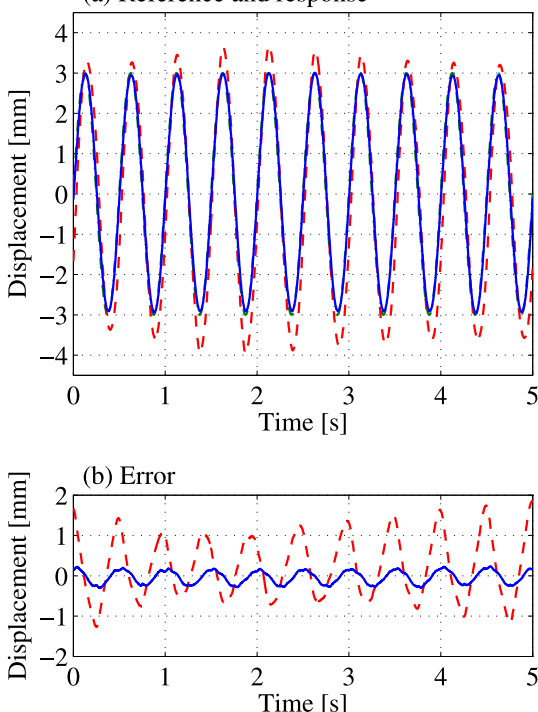

(c) Ref. and res. (Zoomed in 5 times)
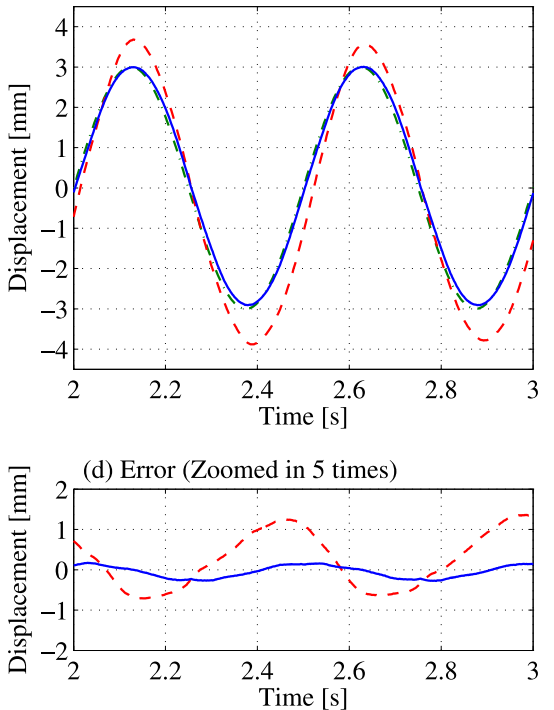

Fig. 14 Excitation results when the water level is set at $130 \mathrm{~mm}$ : (a, c) reference and response signals, and (b, d) error signal. (c) and (d) show signals with 5 times magnification. The reference signal is indicated by the dashed chained line. The response without the compensator is indicated by the dashed red line. The response with the compensator is indicated by the solid blue line.
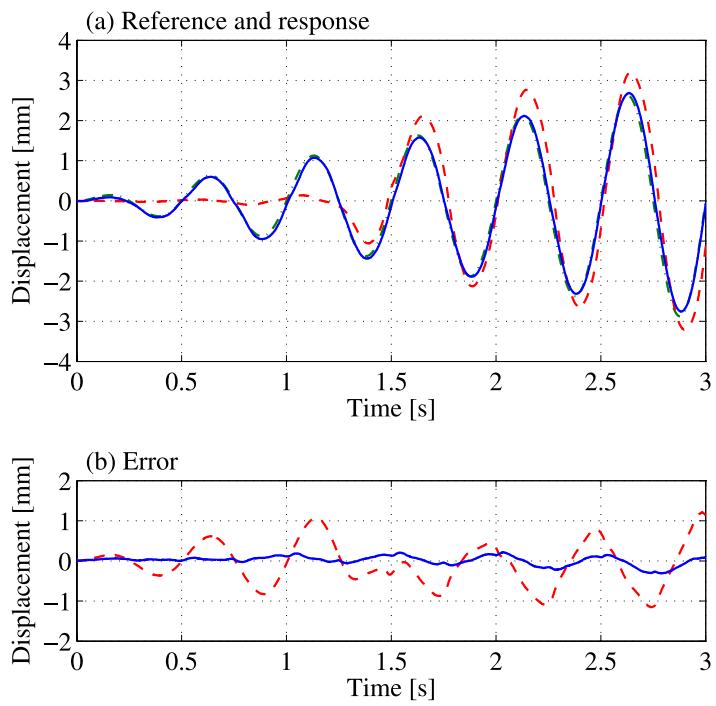

Fig. 15 Transient responses for the influence of friction: (a) reference and response signals, and (b) error signal. The reference signal is indicated by the dashed chained line. The response without the compensator is indicated by the dashed red line. The response with the compensator is indicated by the solid blue line.

\section{Conclusion}

In this paper, a disturbance-force compensator was presented for an electrodynamic shaker. Similar to an actual scenario, a controlled plant with nonlinear characteristics was considered. The control of the shaking system then became difficult. In order to compensate for this problem, the influence of the disturbance force was suppressed. The controller was designed using $\mu$-synthesis by considering the uncertainties of the shaker, and experiments were performed using an electrodynamic shaker with nonlinear characteristics. Because the disturbance-force compensator suppressed the nonlinear characteristic, satisfactory replication of the reference signal was achieved in the control frequency band. These results support the conclusion that the proposed controller is especially useful in this case.

In this study, the perturbation of parameters used in estimating the disturbance-force was 
considered, and the estimator was also added to a generalized plant. However, its effect was not explicitly evaluated in these experiments. Therefore, if parameters of the shaker, which is mainly employed in the estimator, are changed, it will be necessary to confirm the system stability and performance.

\section{References}

( 1 ) Tagawa, Y. and Kajiwara, K., Controller Development for the E-Defense Shaking Table, Proceedings of the Institution of Mechanical Engineers, Part I: Journal of Systems and Control Engineering, Vol.221, No.2(2007), pp.171-181

( 2 ) Yano, K. and Terashima, K., Robust Liquid Container Transfer Control for Complete Sloshing Suppression, IEEE Transactions on Control Systems Technology, Vol.9, No.3(2001), pp.483-493

( 3 ) de Callafon, R., Nagamune, R. and Horowitz, R., Robust Dynamic Modeling and Control of Dual-Stage Actuators, IEEE Transactions on Magnetics, Vol.42, No.2(2006), pp.247-254

( 4 ) Lauwerys, C., Swevers, J. and Sas, P., Robust Linear Control of an Active Suspension on a Quarter Car Test-rig, Control Engineering Practice, Vol.13(2005), pp.577-586

( 5 ) Chida, Y., Kimura, T. and Furukawa, R., Robust Stability Analysis Method for Vibration Systems by using Virtual Perturbations, Proceedings of 2006 IEEE International Conference on Control Applications, (2006-10), pp.1067-1072

( 6 ) Nagashino, T. and Kida, T., Pointing Control of Mobile Antenna on Vehicle, Journal of System Design and Dynamics, Vol.1, No.2(2007), pp.236-244

( 7 ) Uchiyama, Y. and Fujita, M., Robust Multivariable Control of Multi-Axis Electrodynamic Shaking System Using $\mu$-synthesis, Journal of System Design and Dynamics, Vol.2, No.5(2008), pp.1093-1105

( 8 ) Iwasaki, M., Ito, K., Kawafuku, M., Hirai, H., Dozono, Y. and Kurosaki, K., Disturbance observer-based practical control of shaking tables with nonlinear specimen, Proceedings of the 16th IFAC World Congress on Automatic Control, (2005-7)

( 9 ) Seki, K., Iwasaki, M., Kawafuku, M., Hirai, H. and Yasuda, K., Improvement of Control Performance in Shaking-Tables by Feedback Compensation for Reaction Force, Proceedings of the 34th Annual Conference of the IEEE Industrial Electronics Society, (2008-11), pp.2551-2556

(10) Stoten, D. P. and Shimizu, N., The Feedforward Minimal Control Synthesis Algorithm and its Application to the Control of Shaking-Tables, Proceedings of the Institution of Mechanical Engineers, Part I: Journal of Systems and Control Engineering, Vol.221, No.3(2007), pp.423-444

(11) Yi, L., and Tomizuka, M., Two-Degree-of-Freedom Control with Robust Feedback Control for Hard Disk Servo Systems, IEEE/ASME Transactions on Mechatronics, Vol.4, No.1(1999), pp.17-24

(12) Jamaludin, Z., Van Brussel, H. and Swevers, J., Design of a Disturbance Observer and Model-Based Friction Feedforward to Compensate Quadrant Glitches, Motion and Vibration control, (2009), pp.143-154, Springer

(13) Fujita, M., Namerikawa, T., Matsumura, F. and Uchida, K., $\mu$-Synthesis of an Electromagnetic Suspension System, IEEE Transactions on Automatic Control, Vol.40, No.3(1995), pp.530-536 